\title{
Lipid composition and taxonomy of [Pseudomonas] echinoides: transfer to the genus Sphingomonas
}

\author{
Nicola J. Rowe, Jennifer Tunstall, Lesley Galbraith \\ and Stephen G. Wilkinson
}

Author for correspondence: Stephen G. Wilkinson. Tel: +44 1482 651152. Fax: +44 1482466410.

Department of Chemistry, University of Hull, Hull HU6 7RX, UK

\begin{abstract}
Lipid components of [Pseudomonas] echinoides NCIMB 9420 have been studied as an aid to taxonomic relocation of the organism. Non-polar lipids include the carotenoid nostoxanthin and the ubiquinone Q-10. The major fatty acids are cis-vaccenic acid [18:1(11c)], hexadecanoic acid (16:0) and 2-hydroxytetradecanoic acid (2-OH-14:0), but 11-methyloctadec-11-enoic acid [11-Me-18:1(11)] is a significant minor component. The preponderant phospholipids are phosphatidylethanolamine and phosphatidylglycerol; minor lipids include bis(phosphatidyl)glycerol and an unidentified aminophospholipid. Several glycolipids are present, the major one being a glucuronosylceramide derived from sphinganine with amide-bound 2-OH-14:0. The lipid profile supports a proposal to reclassify the organism as Sphingomonas echinoides.
\end{abstract}

Keywords: Pseudomonas echinoides, Sphingomonas, lipids

\section{INTRODUCTION}

[Pseudomonas] echinoides, a little-studied pseudomonad, was first isolated as a laboratory contaminant (Heumann, 1960). The organism is a yellow rod with a single polar flagellum and only the one strain seems to have been deposited in culture collections (ATCC 14820/NCIMB 9420/DSM 1805/ICPB 2835). The organism was left in Section V of Pseudomonas (miscellaneous species) by Palleroni (1984). However, subsequent studies of DNA-rRNA hybridization showed that $[P$.$] echinoides did not belong to the genus$ Pseudomonas as now defined, but it was placed at the base of rRNA superfamily IV (De Vos et al., 1989). Other false pseudomonads in the superfamily (De Vos et al., 1989; Van Bruggen et al., 1993) include [Pseudomonas] pancimobilis, which belongs to the $\alpha-4$ subclass of the Proteobacteria (Takeuchi et al., 1994) and is now the type species of the genus Sphingomonas (Yabuuchi et al., 1990, 1999). A relationship between $[P$.] echinoides and Sphingomonas paucimobilis has also been suggested (Owen \& Jackman, 1982; De Vos et al., 1989) from similarities in DNA base composition, partial DNA-

Abbreviations: DPG, bis(phosphatidyl)glycerol; HPAEC, high-pH anionexchange chromatography; HVE, high-voltage electrophoresis; L-PE, lysophosphatidylethanolamine; PE, phosphatidylethanolamine; PG, phosphatidylglycerol; TMS, trimethylsilyl.
DNA hybridization and the presence in both species of the carotenoid nostoxanthin (Jenkins et al., 1979).

Lipid profiles have found many applications in bacterial chemotaxonomy, including the circumscription of Sphingomonas spp. Distinctive features of these organisms include the absence of 3-hydroxy acids (markers for lipopolysaccharide inter alia), but the presence of 2-hydroxytetradecanoic (2-OH-14:0) and octadecenoic (18:1) acids as major components, together with a range of glycosphingolipids (Yamamoto et al., 1978; Yabuuchi et al., 1979, 1990, 1999; Kawahara et al., 1991; Mizuno et al., 1992; Takeuchi et al., 1993, 1994, 1995; Kämpfer et al., 1997). Characterization of the lipids and fatty acids from $[P$.$] echinoides, the object$ of this study, could therefore clarify the classification of this organism. When this study was almost complete, the results of a detailed chemotaxonomic analysis of $[P$.] echinoides (including lipid data) and a formal proposal to reclassify the species as $S$. echinoides were published (Denner et al., 1999). The results of our own study support and extend these observations.

\section{METHODS}

Organism and growth conditions. Two batches of $[P$. echinoides strain NCIMB 9420 were grown on nutrient agar (Oxoid CM3) for $48 \mathrm{~h}$ at $37^{\circ} \mathrm{C}$ (A) or for $72 \mathrm{~h}$ at $30^{\circ} \mathrm{C}(\mathrm{B})$. Bottles (100), each containing $50 \mathrm{ml}$ medium, were inoculated 
with a suspension of cells washed from starter slopes with sterile nutrient broth (Oxoid CM67). The yields of freezedried, washed cells were $0.84 \mathrm{~g}(\mathrm{~A})$ and $3.0 \mathrm{~g}(\mathrm{~B})$. A further batch of cells (C, yield not determined) was grown on enriched cytophaga solid medium 98 (NCIMB) for $48 \mathrm{~h}$ at $30{ }^{\circ} \mathrm{C}$. S. paucimobilis strain NCTC 11030 was grown on medium 98 for $24 \mathrm{~h}$ at $30^{\circ} \mathrm{C}$ as a source of reference lipids.

Extraction and fractionation of lipids. Lipids were extracted by stirring dry cells with chloroform/methanol $(2: 1, \mathrm{v} / \mathrm{v})$ for $2 \mathrm{~h}$ at room temperature. To avoid possible losses of polar glycolipids, no attempt was made to remove non-lipid contaminants from the extracts. Lipid solutions were stored in the dark at $-20^{\circ} \mathrm{C}$. Fractions soluble in acetone and hexane were used for the examination of carotenoids and isoprenoid quinones, respectively.

Chromatographic and electrophoretic methods. In general, TLC separations were carried out on silica gel $60 \mathrm{~F}_{254}$ (Merck) with the following solvent systems: A, chloroform/ methanol/acetic acid (65:25:10, by vol.); B, chloroform/ methanol/acetic acid/water $(100: 20: 12: 5$, by vol.); C, chloroform/methanol/water $(65: 25: 4$, by vol.); D, chloroform/methanol/acetic acid/water $(80: 12: 15: 4$, by vol.); E, ethyl acetate/pyridine/water (13:5:4, by vol.); F, light petroleum (b.p. $\left.60-80{ }^{\circ} \mathrm{C}\right) /$ acetone $(19: 1, \mathrm{v} / \mathrm{v}) ; \mathrm{G}$, dichloromethane. Aminolipids were detected with ninhydrin, phospholipids with Dittmer-Lester reagent, glycolipids with $\alpha$-naphthol/sulphuric acid and lipids containing 1,2-diol or 1hydroxy-2-amino groups with periodate/Schiff reagents (Christie, 1982). Other methods used to detect lipids were exposure to UV light (for isoprenoid quinones) or iodine vapour, and spraying with ethanolic $2^{\prime}, 7^{\prime}$-dichlorofluorescein. Reverse-phase TLC of isoprenoid quinones was carried out on $\mathrm{RP}-18 \mathrm{~F}_{254}$ plates (Merck) with solvent $\mathrm{H}$ (acetone/ acetonitrile, $4: 1, \mathrm{v} / \mathrm{v})$. HPLC using a $\mathrm{C}_{18}$ column $(25 \mathrm{~cm} \times 4 \cdot 6$ $\mathrm{mm}$ ) eluted with solvent I (methanol/propan-2-ol, $3: 1, \mathrm{v} / \mathrm{v}$ ) and monitored at $270 \mathrm{~nm}$, was also used to identify the major quinone. High-pH anion-exchange chromatography (HPAEC) of monosaccharides utilized a CarboPac PA100 column in a Dionex DX-300 system. Neutral sugars were eluted with $0.016 \mathrm{M} \mathrm{NaOH}$, amino sugars with $0.25 \mathrm{M} \mathrm{NaOH}$ and hexuronic acids with $0.015 \mathrm{M} \mathrm{NaOH}(20 \mathrm{~min})$ followed by a linear gradient $(20 \mathrm{~min})$ to $0 \cdot 15 \mathrm{M}$ sodium acetate $/ 0 \cdot 1 \mathrm{M}$ $\mathrm{NaOH}(10 \mathrm{~min})$.

GLC separations were carried out with either a Carlo Erba Mega 5160 or a Perkin-Elmer Autosystem XL chromatograph, fitted with fused-silica capillary columns ( $25 \mathrm{~m})$ of BP1 (SGE). Finnigan MAT 1020B and GCQ instruments were used for GLC-MS. Paper chromatography and high-voltage electrophoresis (HVE) were carried out with Whatman no. 1 paper and the following solvents and buffers: E; J, ethyl acetate/ pyridine/water/acetic acid $(5: 5: 3: 1$, by vol.); K, pyridine/ acetic acid/water $(5: 2: 43$, by vol., $\mathrm{pH} 5 \cdot 3) ; \mathrm{L}$, pyridine/acetic acid/water $(1: 10: 89$, by vol., adjusted to $\mathrm{pH} 2.7$ with formic acid). Detection reagents used were ninhydrin, aniline hydrogenoxalate, alkaline $\mathrm{AgNO}_{3}$ and the reagent of Hanes \& Isherwood (1949) for phosphate esters.

Studies of polar lipids. Phospholipids were initially characterized by TLC (solvent systems A-D) and by HVE (buffer $\mathrm{K}$ ) at $\mathrm{pH} 5 \cdot 3$ of the water-soluble deacylation products. Confirmation of identity and quantitative data were provided by ${ }^{31} \mathrm{P}$ NMR spectroscopy (London \& Feigenson, 1979), using a JEOL JNM-LA400 spectrometer. Sphingoid bases were released by acid methanolysis (Yano et al., 1982) of total lipids at $60{ }^{\circ} \mathrm{C}$ for $20 \mathrm{~h}$. After extraction of fatty methyl esters with hexane, the hydrolysate was basified $(\mathrm{KOH})$ and re- extracted with hexane/diethyl ether $(1: 1, \mathrm{v} / \mathrm{v})$. The products were examined by TLC (solvent C, detection with ninhydrin and periodate/Schiff reagents) and, as the trimethylsilyl (TMS) derivatives (Yano et al., 1982), by GLC-MS. Analysis for lipid carbohydrate (as glucose) was carried out by a modified phenol/sulphuric acid assay (Kushwaha \& Kates, 1981), and the method of Blumenkrantz \& Asboe-Hansen (1975) was applied for the detection of hexuronic acids. Monosaccharide components of lipids were released by hydrolysis with $2 \mathrm{M}$ trifluoroacetic acid at $98^{\circ} \mathrm{C}$ for $16 \mathrm{~h} \mathrm{(Yu}$ Ip et al., 1992). Neutral sugars were identified by paper chromatography and TLC (both solvent E), HPAEC and GLC of the alditol acetates. The D configuration of glucose was assigned by using D-glucose oxidase (EC 1.1.3.4). Basic and acidic sugars were identified by paper chromatography (solvent J), HVE (buffers $\mathrm{K}$ and L) and HPAEC.

Studies of fatty acids. Fatty acids were released as methyl esters by mild alkaline (Barnes et al., 1989) or acid methanolysis (Yano et al., 1982), or by acid hydrolysis $(6 \cdot 1 \mathrm{M} \mathrm{HCl}$, $100{ }^{\circ} \mathrm{C}, 4 \mathrm{~h}$ ) of the lipids followed by reaction with diazomethane. The products were analysed by GLC and by GLCMS. Saturated and monoenoic esters were separated by argentation TLC (solvent G) and unsaturation was confirmed by hydrogenation. For the location of double bonds, the methyl esters were hydrolysed (Aveldano \& Horrocks, 1983), converted into 3-picolinyl esters (Wait \& Hudson, 1985) and these were also examined by GLC-MS.

Studies of non-polar lipids. Yellow pigments in extracts from $[P]$. echinoides were examined by TLC (solvents A and C) and the visible spectrum of a solution in acetone was recorded with a Philips PU8720 scanning spectrophotometer. The presence of isoprenoid quinones was monitored by TLC (solvent F), reverse-phase TLC and HPLC as described above: material isolated by HPLC was examined by MS.

\section{RESULTS}

Because $[P$.$] echinoides grew poorly in all complex$ liquid media tried, cells for lipid studies were obtained by surface culture. Unpurified lipid extracts constituted $11-12 \%$ of the cell dry weight (batches A and B) and were bright yellow. Two yellow spots (one major, one minor) with high $R_{\mathrm{F}}$ values, greater than those of the most mobile phospholipid, bis(phosphatidyl)glycerol (DPG), were detected by TLC. The visible spectrum obtained for the total acetone-soluble material from batch $\mathrm{A}$, and also for the major, isolated pigment $\left(R_{\mathrm{DPG}}\right.$ $1 \cdot 17$, solvent A), matched that reported for bacterial nostoxanthin (Yabuuchi et al., 1979; Jenkins et al., 1979), consistent with the reported presence of this carotenoid in $S$. paucimobilis and $[P$.] echinoides. Analysis of hexane-soluble products in lipid batch B showed the presence of ubiquinone Q-10, also a distinctive feature of Sphingomonas spp. (Yabuuchi et al., 1999).

Fatty acid profiles for all three batches of lipid were qualitatively similar: the illustrative data shown (Table 1) were determined for lipid batch $B$. The profile obtained after mild alkaline methanolysis was dominated by the methyl esters of just two fatty acids, hexadecanoic acid $(16: 0)$ and a cis-octadecenoic acid $[18: 1(c)]$. The latter, preponderant component was identified as $18: 1$ (11c) (cis-vaccenic acid) by GLC-MS of 
Table 1. Fatty acid composition of the total lipids of $[P$.] echinoides

Data are expressed as percentages of the total peak area on GLC of the methyl esters obtained by mild alkaline methanolysis or acid methanolysis of lipid batch B. TR, Trace.

\begin{tabular}{|lcc|}
\hline Fatty acid* & $\begin{array}{c}\text { Mild alkaline } \\
\text { methanolysis }\end{array}$ & $\begin{array}{c}\text { Acid } \\
\text { methanolysis }\end{array}$ \\
\hline $14: 0$ & TR & TR \\
$15: 0$ & $0 \cdot 5$ & TR \\
$2-\mathrm{OH}-14: 0$ & 0 & $30 \cdot 6$ \\
$16: 1$ & $2 \cdot 2$ & $2 \cdot 2$ \\
$16: 0$ & $23 \cdot 7$ & $23 \cdot 0$ \\
$17: 1$ & $1 \cdot 4$ & TR \\
$17: 0$ & $0 \cdot 7$ & TR \\
$18: 1(11 c)$ & $62 \cdot 2$ & $40 \cdot 1$ \\
$18: 1$ & TR & TR \\
$18: 0$ & $2 \cdot 6$ & TR \\
$11-$ Me-18:1(11) & $6 \cdot 6$ & $3 \cdot 5$ \\
\hline
\end{tabular}

*Shorthand designation. The number before the colon indicates the total number of carbon atoms, the number after the colon the number of double bonds, the number in parentheses the position of the double bond and $c$ the $c i s$ isomer.

the 3-picolinyl ester (Harvey, 1982; Christie et al., 1987). Among the minor components was an unsaturated acid, the methyl ester of which had a GLC retention time a little greater than that of methyl octadecanoate but migrated on argentation TLC just ahead of reference cismonoenoate esters. MS indicated that this was a $\mathrm{C}_{19}$ monoenoic acid (molecular ion with $\mathrm{m} / z$ 310). These properties were consistent with a methyl-branched $18: 1$ acid and MS of the 3-picolinyl ester revealed a spectrum identical to that of the 11-methyloctadec-11-enoic ester from Brevundimonas (Pseudomonas) vesicularis (Wilkinson \& Galbraith, 1979; Barnes et al., 1989). When fatty esters of [P.] echinoides were prepared by acid methanolysis of the lipids, or by acid hydrolysis followed by treatment of the free acids with diazomethane, a third major component was detected and identified as the ester of 2-hydroxytetradecanoic acid (Table 1). Failure to release this component by mild alkaline methanolysis pointed to an amide linkage.

The major phospholipids in extracts from [P.] echinoides were identified by TLC and by HVE of the deacylation products as phosphatidylethanolamine (PE) and phosphatidylglycerol (PG), which accounted for 41 and $32 \%$, respectively, of the lipid phosphorus in batch C (similar proportions were found for batch B). Also apparently present was DPG $(9 \%$ of the lipid phosphorus), although small differences in mobility between the bacterial product and reference lipid were consistently observed by both TLC (e.g. Fig. 1) and HVE. In addition, extracts contained a little $\mathrm{P}_{\mathrm{i}}$ [detected by ${ }^{31} \mathrm{P}$ NMR spectroscopy of the intact lipids (Fig. 2, signal at 2.66 p.p.m.) and by HVE of the deacylation products] and an unidentified aminophospholipid $(15 \%$ of the

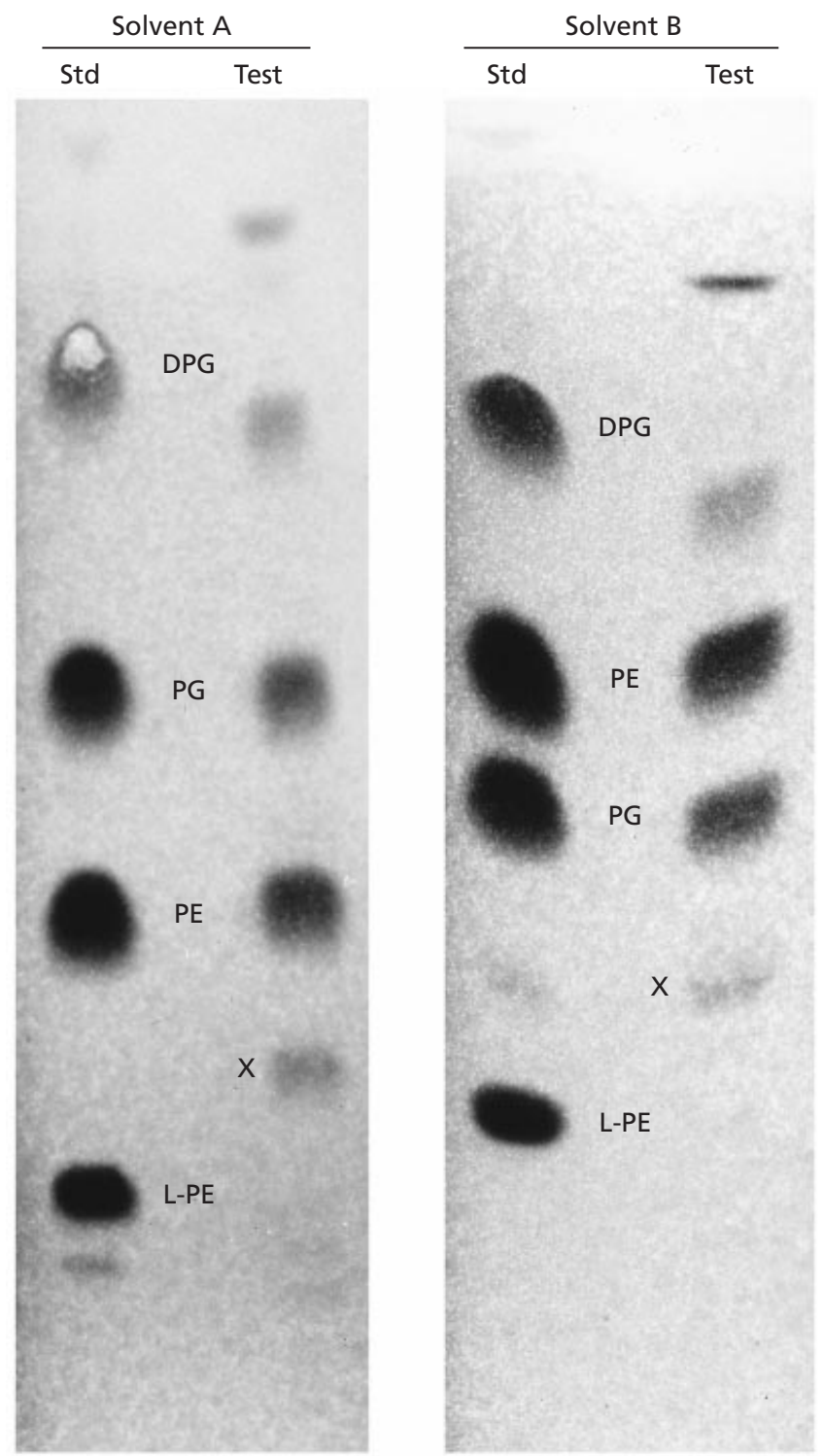

Fig. 1. TLC of the phospholipids of $[P$. $]$ echinoides in solvents $A$ and $B$. Std, reference lipids; Test, lipid batch $C ; X$, unidentified aminophospholipid.

lipid phosphorus). On TLC, the latter had a mobility intermediate between PE and lysophosphatidylethanolamine (L-PE), as illustrated in Fig. 1, and was assumed to give the unattributed signal at 0.64 p.p.m. in the ${ }^{31} \mathrm{P}$ NMR spectrum (Fig. 2). This unidentified lipid was absent from $S$. paucimobilis.

The presence in the lipids of $[P$. $]$ echinoides of amidebound 2-OH-14:0 and the relationship with Sphingomonas spp., prompted a search for (glyco)sphingolipids. After acid methanolysis of the total lipids, a basic, lipophilic product was obtained. On TLC, a spot with the mobility of sphingosine was detected with ninhydrin and periodate/Schiff reagents. The product gave a TMS derivative with a GLC retention time close to that of the sphingosine and GLC-MS showed peaks characteristic 


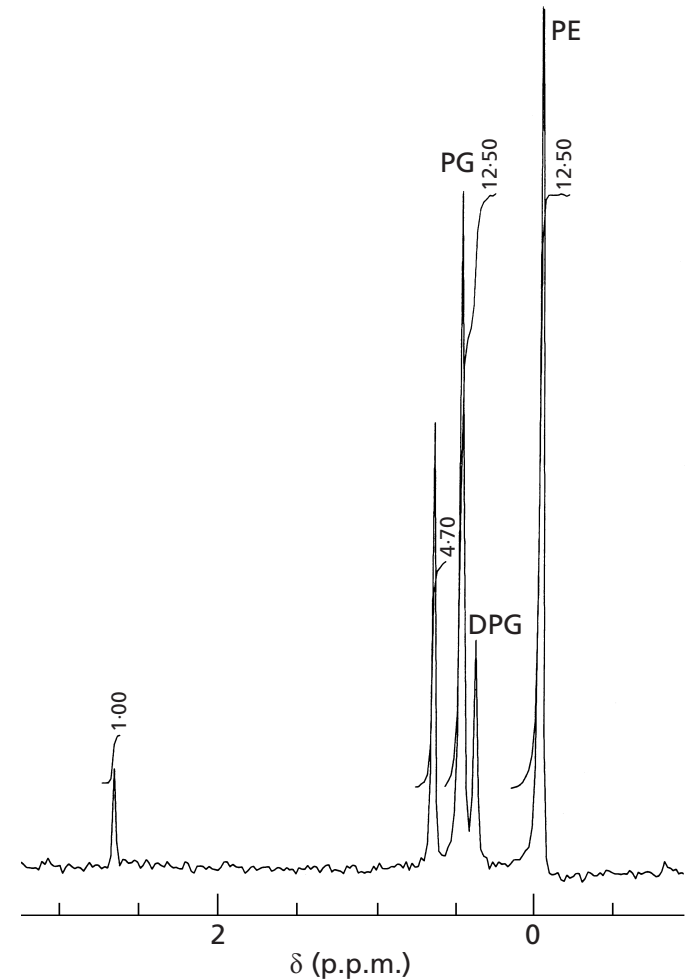

Fig. 2. ${ }^{31} \mathrm{P}$ NMR spectrum of the phospholipids of $[P$. echinoides. Lipids (batch C) were solubilized by the method of London \& Feigenson (1979) and the spectrum was recorded at $162 \mathrm{MHz}$ and ambient temperature with the signal for PE set at 0.00 p.p.m. The signal at 2.66 p.p.m. could be attributed to $P_{i}$ and that at 0.64 p.p.m. was provisionally assigned to the unidentified aminophospholipid X (see Fig. 1).

of the TMS derivatives of sphingoid bases, including the base peak with $m / z 132$ (Yano et al., 1982). Fragment ions with $m / z 430$ [M-15], 342 [M-103] and 313 [M-132] showed that the sphingoid base was a saturated $\mathrm{C}_{18}$ dihydroxyamine, probably 2-aminooctadecane-1,3-diol (dihydrosphingosine or sphinganine).

Colorimetric tests indicated the presence in the lipids of carbohydrate, estimated at $6 \%$ in batch B (expressed as glucose), including a hexuronic acid. Extensive analyses for monosaccharide components showed a difference between the two lipid batches examined (B and C). Both contained glucuronic acid and glucose, but batch B also contained galactose (the major hexose), glucosamine and probably minor amounts of rhamnose and mannose. When $\alpha$-naphthol/sulphuric acid was used to visualize the parent lipids on TLC, several positive reactions were observed. The most intense spot $\left(R_{\mathrm{PE}}\right.$ $\sim 0.4$ in solvent A) matched the major glycolipid in $S$. paucimobilis and was unaffected by mild alkaline methanolysis, consistent with its identification as a glucuronosylceramide (SGL-1; Yabuuchi et al., 1999). Three less intense bands of lower $R_{\mathrm{F}}$ values could correspond to glycosphingolipids produced by further glycosylation and/or non-lipid contaminants. Several other glycolipid bands, with TLC mobilities greater than PE, were detected and could include a monoglycosyldiacylglycerol. Further studies on isolated components are necessary for structural characterization of the unidentified lipids described for $[P$.] echinoides.

\section{DISCUSSION}

This survey of $[P$.$] echinoides has revealed or confirmed$ a distinctive lipid profile for this species. In addition to three common phospholipids (PE, PG and DPG), an unidentified aminophospholipid is a significant component, and one not found in S. paucimobilis. Both organisms also produce glycosphingolipids and the parent ceramide in $[P$. $]$ echinoides apparently has $2-\mathrm{OH}-$ 14:0 amide-linked to the saturated $\mathrm{C}_{18}$ base sphinganine. In the glycosphingolipids of Sphingomonas spp., the same ceramide is accompanied by others containing related sphingoid bases (Yamamoto et al., 1978; Kawahara et al., 1991; Mizuno et al., 1992; Takeuchi et al., 1993, 1994, 1995; Yabuuchi et al., 1999), which might have eluded detection in the present study. Substitution of the ceramides by $\alpha$-D-glucuronic acid produces the simplest glycosphingolipid (SGL-1), further stepwise glycosylation of which leads to a tetrasaccharide derivative (SGL-4) of the following structure: $\alpha$-D-Man $p-(1 \rightarrow 2)-\alpha$-D-Gal $p-(1 \rightarrow 6)-\alpha$-DGlc $p \mathrm{~N}-(1 \rightarrow 4)-\alpha$-D-GlcpA- $(1 \rightarrow 1)$-ceramide (Kawahara et al., 1991; Mizuno et al., 1992). The preliminary data on the monosaccharide composition of lipids from [P.] echinoides suggest a similar extension of SGL-1 in this organism: the failure to detect glucosamine in lipid batch $\mathrm{C}$ could be a consequence of the resistance to acidcatalysed cleavage of the glycosidic bonds formed by hexosamines and hexuronic acids.

Overall, the results of this investigation are in accord with other studies of the carotenoid (Jenkins et al., 1979) and other lipid components (Denner et al., 1999) of [P.] echinoides. In the latter study, the major isoprenoid quinone was identified as Q-10 and the principal fatty acids as $2-\mathrm{OH}-14: 0,16: 0$ and $18: 1$. Based on the results of two-dimensional (2D) TLC, the major phospholipids were reported as PE and PG; other phospholipids found included an unidentified aminophospholipid and a spot corresponding to SGL-1 was visualized. No further characterization of the polar lipids was apparently attempted, no information on sphingoid base nor monosaccharide composition was presented and the 11Me-18:1(11) fatty acid was not described. On the other hand, the use of 2D-TLC provided enhanced resolution of the polar lipids and detection of several other unidentified (glyco/phospho)lipids. Both the results of Denner et al. (1999) and the present study support the transfer of $[P$.$] echinoides to the genus Sphingomonas.$ Within the genus there is some diversity of lipid composition, e.g. $[P$.$] echinoides lacks the methylated$ derivatives of PE (including phosphatidylcholine) found in other Sphingomonas spp. (Kämpfer et al., 1997; Denner et al., 1999) and there can be significant variations in fatty acid composition. The preponderant 18:1 acid in several species has been listed as $18: 1(9)$ by 
Yabuuchi et al. (1999) in contrast to $18: 1(11 c)$ found here in $[P$.$] echinoides. In other studies, the relative$ abundance of individual members of the dominant envelope of GLC peaks [18:1(6t), 18:1(9t), 18:1(11c)] was not reported (Kämpfer et al., 1997; Denner et al., 1999). The fatty acid provisionally identified here as 11-Me-18:1(11) has not been found in other Sphingomonas spp. but, interestingly, it (or another methyl-branched monoenoic acid) has been described for the lipids from other organisms ( $R h i$ zobium, Bradyrhizobium, Brevundimonas, Caulobacter and Hyphomonas spp.) belonging to the $\alpha$-subclass of the Proteobacteria (Gerson et al., 1975 ; Gerson \& Patel, 1975; MacKenzie et al., 1979; Andreev et al., 1986; Barnes et al., 1989; Abraham et al., 1997, 1999).

\section{REFERENCES}

Abraham, W.-R., Meyer, H., Lindholst, S., Vancanneyt, M. \& Smit, J. (1997). Phospho- and sulfolipids as biomarkers of Caulobacter sensu lato, Brevundimonas and Hyphomonas. Syst Appl Microbiol 20, 522-539.

Abraham, W.-R., Strömpl, C., Meyer, H. \& 8 other authors (1999). Phylogeny and polyphasic taxonomy of Caulobacter species. Proposal of Maricaulis gen. nov. with Maricaulis maris (Poindexter) comb. nov. as the type species, and emended description of the genera Brevundimonas and Caulobacter. Int J Syst Bacteriol 49, 1053-1073.

Andreev, L. V., Akimov, V. N. \& Nikitin, D. I. (1986). Peculiarities of fatty acid composition of the genus Caulobacter. Folia Microbiol 31, 144-153.

Aveldano, M. I. \& Horrocks, L. A. (1983). Quantitative release of fatty acids from lipids by a simple hydrolysis procedure. J Lipid Res 24, 1101-1105.

Barnes, A., Galbraith, L. \& Wilkinson, S. G. (1989). The presence of 11-methyloctadec-11-enoic acid in the extractable lipids of Pseudomonas vesicularis. FEMS Microbiol Lett 59, 101-106.

Blumenkrantz, N. \& Asboe-Hansen, G. (1975). New method for quantitative determination of uronic acids. Anal Biochem 54, 484-489.

Christie, W. W. (1982). Lipid Analysis, 2nd edn. Oxford: Pergamon.

Christie, W. W., Brechany, E. Y. \& Holman, R. T. (1987). Mass spectra of the picolinyl esters of isomeric mono- and dienoic fatty acids. Lipids 22, 224-228.

Denner, E. B. M., Kämpfer, P., Busse, H.-J. \& Moore, E. R. B. (1999). Reclassification of Pseudomonas echinoides Heumann 1962, 343 ${ }^{\mathrm{AL}}$, in the genus Sphingomonas as Sphingomonas echinoides comb. nov. Int J Syst Bacteriol 49, 1103-1109.

De Vos, P., van Landschoot, A., Segers, P. \& 9 other authors (1989). Genotypic relationships and taxonomic localization of unclassified Pseudomonas and Pseudomonas-like strains by deoxyribonucleic acid: ribosomal ribonucleic acid hybridizations. Int J Syst Bacteriol 39, 35-49.

Gerson, T. \& Patel, J. J. (1975). Neutral lipids and phospholipids of free-living and bacteroid forms of two strains of Rhizobium infective to Lotus pedunculatus. Appl Microbiol 30, 193-198.

Gerson, T., Patel, J. J. \& Nixon, N. L. (1975). Some unusual fatty acids of Rhizobium. Lipids 10, 134-139.

Hanes, C. S. \& Isherwood, F. A. (1949). Separation of the phosphoric esters on the filter paper chromatogram. Nature 164, 1107-1112.
Harvey, D. J. (1982). Picolinyl esters as derivatives for the structural determination of long chain branched and unsaturated fatty acids. Biomed Mass Spectrom 9, 33-38.

Heumann, W. (1960). Versuche zur Rekombination sternbildender Bakterien. Naturwissenschaften 47, 330-331.

Jenkins, C. L., Andrewes, A. G., McQuade, T. J. \& Starr, M. P. (1979). The pigment of Pseudomonas paucimobilis is a carotenoid (nostoxanthin), rather than a brominated aryl-polyene (xanthomonadin). Curr Microbiol 3, 1-4.

Kämpfer, P., Denner, E. B. M., Meyer, S., Moore, E. R. B. \& Busse, H.-J. (1997). Classification of 'Pseudomonas azotocolligans' Anderson 1955, 132, in the genus Sphingomonas as Sphingomonas trueperi sp. nov. Int J Syst Bacteriol 47, 577-583.

Kawahara, K., Seydel, U., Matsuura, M., Danbara, H., Rietschel, E. T. \& Zähringer, U. (1991). Chemical structure of glycosphingolipids isolated from Sphingomonas paucimobilis. FEBS Lett 292, 107-110.

Kushwaha, S. C. \& Kates, M. (1984). Modification of phenolsulfuric acid method for the estimation of sugars in lipids. Lipids 16, 372-373.

London, E. \& Feigenson, G. W. (1979). Phosphorus NMR analysis of phospholipids in detergents. J Lipid Res 20, 408-412.

MacKenzie, S. L., Lapp, M. S. \& Child, J. J. (1979). Fatty acid composition of Rhizobium spp. Can J Microbiol 25, 68-74.

Mizuno, S., Hotta, H., Yabuuchi, E. \& Yano, I. (1992). FAB/MS analysis of novel sphingoglycolipids in Sphingomonas species. Nippon Iyo Masu Supekutoru Gakkai Koenshu 17, 179-182.

Owen, R. J. \& Jackman, P. J. H. (1982). The similarities between Pseudomonas paucimobilis and allied bacteria derived from analysis of deoxyribonucleic acids and electrophoretic protein patterns. J Gen Microbiol 128, 2945-2954.

Palleroni, N. (1984). Genus Pseudomonas Migula $1893^{\mathrm{AL}}$. In Bergey's Manual of Systematic Bacteriology, vol. 1, pp. 141-199. Edited by N. R. Krieg \& J. G. Holt. Baltimore: Williams \& Wilkins.

Takeuchi, M., Kawai, F., Shimada, Y. \& Yokota, A. (1993). Taxonomic study of polyethylene glycol-utilizing bacteria: emended description of the genus Sphingomonas and new descriptions of Sphingomonas macrogoltabidus sp. nov., Sphingomonas sanguis sp. nov. and Sphingomonas terrae sp. nov. Syst Appl Microbiol 16, 227-238.

Takeuchi, M., Sawada, H., Oyaizu, H. \& Yokota, A. (1994). Phylogenetic evidence for Sphingomonas and Rhizomonas as nonphotosynthetic members of the alpha-4 subclass of the Proteobacteria. Int J Syst Bacteriol 44, 308-314.

Takeuchi, M., Sakane, T., Yanagi, M., Yasamato, K., Hamana, K. \& Yokota, A. (1995). Taxonomic study of bacteria isolated from plants: proposal of Sphingomonas rosa sp. nov., Sphingomonas pruni sp. nov., Sphingomonas asaccharolytica sp. nov., and Sphingomonas mali sp. nov. Int J Syst Bacteriol 45, 334-341.

Van Bruggen, A. H. C., Jochimsen, K. N., Steinberger, E. M., Segers, P. \& Gillis, M. (1993). Classification of Rhizomonas suberifaciens, an unnamed Rhizomonas species, and Sphingomonas spp. in rRNA superfamily IV. Int J Syst Bacteriol 43, 1-7.

Wait, R. \& Hudson, M. J. (1985). The use of picolinyl esters for the characterization of microbial lipids: application to the unsaturated and cyclopropane fatty acids of Campylobacter species. Lett Appl Microbiol 1, 95-99.

Wilkinson, S. G. \& Galbraith, L. (1979). Polar lipids of Pseudomonas vesicularis. Presence of a heptosyldiacylglycerol. Biochim Biophys Acta 575, 244-254.

Yabuuchi, E., Tanimura, E., Ohyama, A., Yano, I. \& Yamamoto, A. 
(1979). Flavobacterium devorans ATCC 10829: a strain of Pseudomonas paucimobilis. J Gen Appl Microbiol 25, 95-107.

Yabuuchi, E., Yano, I., Oyaizu, H., Hashimoto, Y., Ezaki, T. \& Yamamoto, H. (1990). Proposals of Sphingomonas paucimobilis gen. nov. and comb. nov., Sphingomonas parapaucimobilis sp. nov., Sphingomonas yanoikuyae sp. nov., Sphingomonas adhaesiva sp. nov., Sphingomonas capsulata comb. nov., and two genospecies of the genus Sphingomonas. Microbiol Immunol 34, 99-119.

Yabuuchi, E., Kosako, Y., Naka, T., Suzuki, S. \& Yano, I. (1999). Proposal of Sphingomonas suberifaciens (van Bruggen, Jochimsen and Brown 1990) comb. nov., Sphingomonas natatoria (Sly 1985) comb. nov., Sphingomonas ursincola (Yurkov et al., 1997) comb. nov., and emendation of the genus Sphingomonas. Microbiol Immunol 43, 339-349.
Yamamoto, A., Yano, I., Masui, M. \& Yabuuchi, E. (1978). Isolation of a novel sphingoglycolipid containing glucuronic acid and 2-hydroxy fatty acid from Flavobacterium devorans ATCC 10829. J Biochem (Tokyo) 83, 1213-1216.

Yano, I., Tomiyasu, I. \& Yabuuchi, E. (1982). Long chain base composition of strains of three species of Sphingobacterium gen. nov. FEMS Microbiol Lett 15, 303-307.

Yu Ip, C. C., Manam, V., Hepler, R. \& Hennessey, J. P. (1992). Carbohydrate composition analysis of bacterial polysaccharides: optimized acid hydrolysis conditions for HPAEC-PAD analysis. Anal Biochem 201, 343-349.

Received 8 March 2000; revised 26 June 2000; accepted 11 July 2000. 